\title{
Relative roles of spatial and intensive cues in the discrimination of spatial tactile stimuli
}

\author{
GREGORY O. GIBSON and JAMES C. CRAIG \\ Indiana University, Bloomington, Indiana
}

\begin{abstract}
Two psychophysical measures of tactile sensitivity - grating-orientation (GO) and smooth-grooved (SG) discrimination-were used to determine tactile spatial acuity with and without an intermediate surface (latex glove) interposed between the contactor and the skin. Measures were made at three locations that varied in sensitivity and in density of innervation of the primary afferent fibers: the index fingerpad (fingertip), palmar surface of the proximal phalanx (fingerbase), and the thenar eminence (palm). Neurophysiological studies have suggested that the density of innervation of SAI fibers is a limiting factor in spatial acuity. In the present study, without a glove, the GO thresholds varied as a function of location. With the glove, increases in the GO thresholds were relatively uniform and modest. Without a glove, however, the SG thresholds were well below the GO thresholds, and changing the site of stimulation had little effect on the threshold. With a glove, the SG thresholds increased by $100 \%$ to more than $300 \%$ as in comparison with those in the no-glove condition. The largest increases occurred at the less sensitive locations. The results of the GO task are consistent with the view that GO is a valid measure of spatial acuity. The results of the SG task, however, are inconsistent with previous results and suggest that both spatial and intensive factors are involved in this task.
\end{abstract}

Much of the tactile information that we receive is transmitted through intermediate surfaces, such as tools or clothing (especially gloves). In the present study, we examined the effect of surgical gloves on tactile sensitivity and the differential effects that this intermediate surface has on two measures of tactile sensitivity. Studies on the effects of gloves on the sense of touch have been motivated by both applied and theoretical interests. Particularly with regard to surgical gloves, there has been a continuing interest in providing protection for both the surgeon and the patient and, at the same time, maintaining adequate tactile sensitivity. In addition, remote sensing and teleoperator systems must rely on receiving tactile information through intermediate surfaces (Klatzky \& Lederman, 1999). An understanding of the effects of gloves on tactile sensitivity should suggest what aspects of tactile information need to be preserved in order to provide effective remote sensing. On the theoretical side, comparing performance with and without gloves may increase our understanding of sensory measures and sensory mechanisms.

Of the various measures of tactile sensitivity that are available, we selected spatial acuity for several reasons. First, it is an important component of tactile sensitivity. Changes

This research was supported by the National Institutes of Health Grant DC 00095, National Institute on Deafness and Other Communication Disorders. The authors thank Roger Rhodes for his assistance in these experiments and Dwight Hector for his technical assistance in designing and building the contactors used in making the penetration measurements. Correspondence should be addressed to G. O. Gibson, Department of Psychology, Indiana University, Bloomington, IN 47405 (e-mail: gogibson@indiana.edu). in spatial acuity affect our ability to judge roughness, identify objects, and discriminate surfaces from one another, among other things. Second, our understanding of the processing of spatial information has advanced considerably in recent years, due in large measure to the close relationship between psychophysical and neurophysiological studies of spatial mechanisms (Johnson \& J. R. Phillips, 1981; J. R. Phillips \& Johnson, 1981a, 1981b). Knowing how spatial information is processed in the absence of gloves should allow for a greater understanding of the possible effects, if any, that gloves have on spatial acuity.

Because spatial sensitivity is an important tactile function, it is not surprising that a number of studies have examined the effect that gloves have on it. These studies have used a wide variety of tasks that may involve spatial mechanisms. In some of these studies, no significant decrease in spatial sensitivity with gloves was reported (Brisben, Hsiao, Looft, \& Johnson, 1992; Heller \& Mitchell, 1985; Klatzy \& Lederman, 1999; Nelson \& Mital, 1995; Novak, Patterson, \& Mackinnon, 1999; Thompson \& Lambert, 1995; Webb \& Pentlow, 1993; M. P. Wilson, Gound, Tishk, \& Feil, 1986), whereas, in other studies, gloves significantly reduced spatial sensitivity (Cunningham, Delargy, \& Warnock, 1992; A. M. Phillips, Birch, \& Ribbans, 1997). Part of the difficulty in drawing a definitive interpretation from these studies is due to the wide variety of measures used and the fact that these studies were not defining tactile sensitivity in the same way. Some of the studies used tasks such as roughness discrimination and texture matching, although it should be noted that, with regards to roughness, cues other than spatial cues may play a role (Stevens, 1990). Other investigators have used a tra- 
ditional measure of spatial sensitivity, such as the twopoint threshold. In the studies that used the two-point threshold, no change in sensitivity with gloves was found (Novak et al., 1999; Thompson \& Lambert, 1995; Webb \& Pentlow, 1993); however, there are a number of problems with the two-point threshold as a measure of spatial acuity. Chief among these problems is the unreliability of the results both between and within subjects (Craig \& Johnson, 2000; Johnson, Van Boven, \& Hsiao, 1994).

In recent psychophysical studies, some done in conjunction with neurophysiological studies, two relatively new measures of spatial acuity have been used: grating orientation (GO) (Johnson \& J. R. Phillips, 1981) and gap detection (Stevens \& Choo, 1996; Stevens \& Patterson, 1995). The GO measure involved presenting square-wave gratings to the skin. The gratings were cut into circular domed contactors. The contactors were presented to the skin in one of two orientations, one at $90^{\circ}$ to the other. The subject's task was to identify the orientation. Accuracy is measured as a function of the width of the grooves. GO as a psychophysical measure of spatial acuity has been measured at a number of different body loci, which vary in their densities of innervation and sensitivity (Craig, 1999; Craig \& Kisner, 1998; Craig \& Lyle, 2001; Essock, Krebs, \& Prather, 1997; Johnson \& J. R. Phillips, 1981; Patel, Essick, \& Kelly, 1997; Sathian \& Zangaladze, 1996; Van Boven \& Johnson, 1994a). The second task, gap detection, required a subject to distinguish between a surface or an edge that was smooth and one that had one or more grooves or gaps in it. It, too, has been tested at a number of different body loci and with subjects of different ages (Stevens \& Choo, 1996; Stevens \& Patterson, 1995).

Psychophysical and neurophysiologicalstudies suggest that spatial acuity as measured by GO changes with the density of innervation and, thus, the spacing of the peripheral receptors (Craig, 1999; Johnson \& J. R. Phillips, 1981; Van Boven \& Johnson, 1994a). There are two types of peripheral afferents that appear to convey spatial information: rapidly adapting (RA) and slowly adapting Type I (SAI) afferents. Of the two, SAI afferents convey the finest spatial information (Johnson \& Hsiao, 1994). For the GO task, a groove width of approximately $1 \mathrm{~mm}$ yields a threshold performance level of $75 \%$ correct (in a twoalternative, forced-choice procedure) on the fingertip. This threshold value corresponds to the estimated density of innervation of one SAI afferent per square millimeter and spacing between receptors of approximately $1 \mathrm{~mm}$ (Johnson \& Hsiao, 1992; Johnson \& Phillips, 1981). Neurophysiological results show a sharp decline in the density of innervation as one moves proximally from the fingertip (Johansson \& Vallbo, 1979). Paralleling this decline, the threshold for GO increases from approximately $1 \mathrm{~mm}$ to approximately $2 \mathrm{~mm}$ as one moves from a test site $15 \mathrm{~mm}$ proximal to the fingertip to a site $25 \mathrm{~mm}$ proximal to the fingertip (Craig, 1999).

Van Boven and Johnson (1994b) also found evidence that sensitivity to GO is closely related to the underlying neural mechanisms. In their study, they made a series of psychophysical measurements during recovery following injury of the trigeminal nerve. Many of the psychophysical measures of tactile sensitivity, such as the two-point threshold, direction of brush stroke, pressure sensitivity, and so forth, returned to normal sensitivity levels long before self-reports of normal tactile sensitivity. It took much longer (6 months to 1 year) for GO performance to return to preinjury levels. Performance on the GO task and its return to preinjury levels were consistent with subjects' selfreports of normal sensory function and its return. The time course of full recovery of sensitivity in the GO task was also consistent with the rate of reinnervation by peripheral afferent fibers (Van Boven \& Johnson, 1994b). In short, spatial acuity as measured by GO appears to be correlated with the density of innervation.

As a second measure of spatial sensitivity, we used a variation of a gap detection measure. In one of the early versions of this task, subjects touched the edge of a disk, $2.0 \mathrm{~mm}$ in width. The disk either was smooth or had a gap cut into it. The subject's task was to indicate whether the disk was smooth (no gap) or had a gap in it. In general, performance varied as a function of the size of the gap, with larger gaps being easier to detect than smaller ones (Johnson \& J. R. Phillips, 1981). Stevens and colleagues used a variation of this task in several studies (Stevens \& Choo, 1996; Stevens \& Patterson, 1995). In this version, subjects were presented with one of two edges, one of which contained a gap. The subject's task was to indicate which edge contained the gap. Stevens's results have shown that sensitivity, as measured by this technique, varies as a direct function of gap size. Still another variation of the gap detection task was used by investigatorsto examine anisotropies in the human tactile system (Essock et al., 1997; Wheat \& Goodwin, 2000). In these studies, subjects were required to distinguish between a flat smooth contactor and a flat contactor with one or more grooves cut into it. Craig (1999) used a modified version of these tasks. In this version, which Craig referred to as a smooth/grooved task (SG), subjects were presented with a smooth domed contactor or a domed contactor that had multiple, equal-width grooves and ridges. These were the same contactors used in the GO task. The subject's task was to identify the contactor as either smooth or grooved. Performance was measured as a function of groove width.

Recordings from first-order afferents have been made in response to the surface of the skin being presented with grooves of various widths, including smooth surfaces. These recordings show how certain types of afferents respond to the various groove widths and how spatial information is encoded peripherally (J. R. Phillips \& Johnson, 1981a). One of the reasons for using the GO and SG tasks in the present study was that the stimuli used in these tasks were similar to the stimuli used in the physiological recording work. This similarity made it easier to relate psychophysical results to the neurophysiologicalresults and spatial and intensive encoding mechanisms. In discussing 
spatial and intensive mechanisms, we are relying on the distinctions that Johnson and J. R. Phillips (1981, p.1178) made: An intensive mechanism "depends on the impulse rates in single fibers but makes no use of the exact location of the afferent terminals ... A mechanism is purely spatial if it depends on the exact locations of active neurons but is unaffected by uniform changes in impulse rate."

If the SG task is tapping spatial mechanisms, then performance on this task should vary in the same way as performance on the GO task. In general, Johnson and J. R. Phillips (1981) found that performance on their gap detection task paralleled other measures of spatial acuity. They noted, however, that, at narrow gaps (less than $0.7 \mathrm{~mm}$ ), performance on the SG task was above chance and independent of groove width. They suggested that two different mechanisms might be responsible for the SG task: Larger groove widths might be tapping spatial mechanisms, whereas smaller groove widths might be encoded by changes in intensity. Even if the SG task is not completely correlated with other measures of spatial acuity, it may reflect an important perceptual capability, discriminating between smooth and grooved surfaces. One might imagine that if the gloves interfered with the ability to make such a discrimination, this interference would contribute to the feeling that gloves distort or alter the sense of touch.

Because of their sensitivity, the fingertips have been the site of most of the testing of spatial acuity on the hand, including testing the effects of gloves. The fingertips are also likely to be used in haptic exploration. It has been hypothesized that, with an appropriate adjustment of spatial frequencies, the processing of spatial information should be similar across regions that differ in sensitivity (Loomis \& Lederman, 1986). Recent measurements of spatial acuity support this hypothesis. Using the GO task, similar psychometric functions were obtained on the fingertip and palm. Relative to the fingertip, the palm function was shifted to lower spatial frequencies. The width of gratings had to be seven to nine times wider on the palm to produce the same levels of performance as those obtained testing the fingertip (Craig \& Lyle, 2001).

The alteration in sensitivity reported by most glove wearers may reflect changes at more than just the fingertips; however, few of the previous studies with gloves have measured sensitivity at multiple sites on the hand. If gloves reduce spatial acuity at the fingertips, do they also reduce sensitivity by a corresponding amount at different sites? Does a glove produce an effect similar to testing at a less densely innervated site? If gloves produce differential changes in sensitivity across areas, it will be necessary to make measurements at several sites on the hand to assess the impact of gloves on tactile sensitivity. Measuring sensitivity at several sites also permits us to compare relative changes in the GO and SG tasks.

The present study consisted of three experiments. In Experiment 1, GO sensitivity was measured at three sites on the hand with and without a glove. In Experiment 2, SG sensitivity was measured at the same three sites on the hand with and without a glove. In Experiment 3, additional GO measurements were made at the fingerbase, as were measures of the penetration of the skin into the grooves of the contactors.

\section{EXPERIMENT 1}

GO sensitivity was measured at the fingertip, the fingerbase, and the palm.

\section{Method}

Subjects. The subjects were students at Indiana University who were paid employees of the laboratory. The number of subjects tested in each condition is given in Table 1.

Stimuli. The stimuli were domed contactors with square-wave gratings cut into them. These contactors (JVP Domes) are commercially made by Stoelting Co. (Wood Dale, IL). Each contactor had a series of equal-width grooves and ridges. The grooves were cut sufficiently deep so that the skin did not touch the bottom of the groove. The contactors were $19 \mathrm{~mm}$ in diameter. Each set consisted of eight commercially made contactors, with groove widths of $3,2,1.5,1.2$, $1,0.75,0.50$, and $0.35 \mathrm{~mm}$. In several conditions, thresholds sometimes exceeded $3 \mathrm{~mm}$. For this reason, additional contactors were machined with larger grooves $(6,5,4 \mathrm{~mm})$. These three additional contactors were also $19 \mathrm{~mm}$ in diameter. In addition to these contactors, a new set of contactors was machined for testing GO on the palm. The new set consisted of seven contactors, with groove widths of $12,10,8,6,4,2$, and $1 \mathrm{~mm}$. To accommodate the larger groove widths, the contactors used on the palm were $50 \mathrm{~mm}$ in diameter.

Apparatus. A counterweighted lever was used to present the contactors with a 100-gm force. An air shock was used to control and smooth the travel of the lever arm to which the contactor was attached. Because of differences in the size of the contactors, a 190gm force was used when the palm was the test site in the GO conditions. The intent was to make the depth of penetration approximately the same at the three test sites. The surgical gloves were "The Original Perry Style 42 White Latex Surgical Glove" manufactured by Ansell Perry, Inc. (Massillon, $\mathrm{OH}$ ). These latex surgical gloves came in fitted sizes ranging from $51 / 2-9$. The gloves were $0.20-\mathrm{mm}$ thick.

Procedure. The subjects were tested individually. They were randomly assigned to start each condition either with or without a glove

Table 1

Number of Subjects Tested in Each Condition of Experiments 1, 2, and 3

\begin{tabular}{|c|c|c|c|c|c|c|}
\hline \multirow[b]{2}{*}{ Location } & \multicolumn{2}{|c|}{ Experiment $1(\mathrm{GO})$} & \multicolumn{2}{|c|}{$\begin{array}{c}\text { Experiment } 2 \\
\text { (Smooth Groove) } \\
\end{array}$} & \multicolumn{2}{|c|}{$\begin{array}{l}\text { Experiment } 3 \\
\text { (Oblique GO) }\end{array}$} \\
\hline & Male & Female & Male & Female & Male & Female \\
\hline Distal pad & 1 & 7 & 2 & 6 & n.a. & n.a. \\
\hline Proximal pad & 1 & 6 & 3 & 5 & 3 & 4 \\
\hline Palm & 1 & 7 & 3 & 7 & n.a. & n.a. \\
\hline
\end{tabular}

Note-GO, grating orientation; n.a., not available. 
covering the test site. Thereafter, they alternated sessions between testing with or without a glove. Each of the three locations on the right hand - namely, the index fingerpad (fingertip), the palmar surface of the proximal phalanx (fingerbase), and the palm (thenar eminence) - were tested over six sessions (three with glove, three without glove). During each testing session, the subject was seated with his/her right arm extended with the medial edge of the palm and forearm resting on the table. Depending on the site to be tested, the subject's hand or finger was positioned on a padded shelf so the contactor could be brought in contact with the test site. The subjects were instructed not to move their hands or fingers during testing. Each trial began with the experimenter presenting the contactor and ended with the subjects' response. The contactor remained in contact with the subjects' skin until they responded (approximately 1$2 \mathrm{sec}$ ), at which time the experimenter removed the contactor. During testing, the subjects kept their eyes closed.

The subjects were instructed that a grooved contactor would be presented in one of two orthogonal orientations (proximal-distal or lateral-medial). The two orientations were explained to the subjects. On each trial, they were to respond with "proximal" or "lateral." Each orientation was demonstrated, while the subject observed the presentation. The subjects then received several practice trials, during which they kept their eyes closed. Every session began with practice trials with feedback; however, during testing, no feedback was provided.

A session consisted of six blocks of 30 trials, for a total of 180 trials per session. On the index fingerpad, the contactor was presented to the center of the fingertip $15 \mathrm{~mm}$ proximal to the tip of the finger. Each session started with the first block, using a groove width of $3 \mathrm{~mm}$. Successive blocks of trials tested smaller groove widths in the following order: $2,1.5,1,0.75$, and $0.35 \mathrm{~mm}$. On the fingerbase, the contactor was presented midway between the two joints (proximal interphalangeal and metacarpophalangeal). Each session started with the first block, using a groove width of $6 \mathrm{~mm}$. Each successive block of trials tested a smaller groove width: 5, 4, 3, 2, and $1 \mathrm{~mm}$. On the palm, the center of the thenar eminence was tested. Each session started with the first block, using a groove width of $12 \mathrm{~mm}$. Each successive block of trials tested a smaller groove width: 10, 8 , 6,4 , and $2 \mathrm{~mm}$. The orientation (proximal vs. lateral) of the contactor on each trial was determined randomly.

\section{Results}

The results for the three sites are plotted in Figure 1, percent correct as a function of the log of the groove width. It appears that the glove produced a decline in sensitivity at all three test sites. A repeated measures analysis of variance (ANOVA) was conducted to see whether there was a significant difference between the glove condition and the no-glove condition. The glove had a significant effect on performance at the fingertip, fingerbase, and palm $[F(1,7)=$ $13.18, F(1,6)=9.66$, and $F(1,7)=17.48$, respectively, $p$ s $<.05]$. As expected, there was a significant effect of groove width at the fingertip, fingerbase, and palm $[F(5,35)=97.72, F(5,30)=44.36$, and $F(5,35)=65.81$, respectively, $p \mathrm{~s}<.01]$. At the fingertip, there was a significant interaction between glove condition and groove width $[F(5,35)=4.03, p<.01]$. This interaction appears to have been due to the convergence of the functions at or near chance and at or near $100 \%$ correct.

In this study, $75 \%$ correct (halfway between chance and $100 \%$ correct) was adopted as a threshold measure (Johnson \& J. R. Phillips, 1981). For the results presented in Figure 1, the threshold was calculated by linear interpola- tion. The threshold values for the GO task with and without gloves, along with the percentage change in sensitivity, are presented in Table 2. As can be seen, there was a modest increase in the thresholds in the glove condition.

\section{Discussion}

A number of studies have measured the GO threshold at the fingertip. The threshold values have ranged from $0.94 \mathrm{~mm}$ (Johnson \& J. R. Phillips, 1981) to $1.36 \mathrm{~mm}$ (Craig \& Lyle, 2001). The threshold obtained without a glove in the present experiment $(1.24 \mathrm{~mm})$ was within the range of these previous studies. For the fingerbase, there was one earlier study that measured GO sensitivity. The threshold was $4.80 \mathrm{~mm}$ (Craig, 1999), again similar to the threshold of $4.35 \mathrm{~mm}$ in the present experiment. For the palm, the threshold was somewhat lower $(5.73 \mathrm{~mm})$ than that obtained in an earlier study, in which the thresholds varied from 7.8 to $9.2 \mathrm{~mm}$ (Craig \& Lyle, 2001). The test site in the earlier study was the center of the palm; in the present experiment, the test site was the thenar eminence. It is likely that the thenar eminence, associated as it is with the base of the thumb, has a greater density of innervation than the center of the palm and would be more sensitive to spatial stimuli than the center of the palm.

As noted, Loomis and Lederman (1986) suggested that spatial information should be processed similarly at different sites on the skin. If that is the case, the resulting psychometric functions should be similar to one another. In fact, the functions should be parallel to one another when performance is plotted as the log of the spatial dimension. The no-glove function on the fingerbase, however, appears to be somewhat different from the other functions in that it appears to have two distinct limbs. At the smaller groove widths (Figure 1, fingerbase, no glove), performance did not decline as rapidly as would be predicted by extrapolating from the function obtained at the larger groove widths. At the larger groove widths, the function paralleled the functions obtained at the other locations. It may be that, at the larger groove widths, the task was being done on the basis of spatial information. At smaller groove widths, as suggested by Johnson and J. R. Phillips (1981), it may have been done on the basis of intensive information. A possible reason for these differences and the role of intensity cues in the GO task on the fingerbase are considered in Experiment 3 (presented later).

Density of innervation of the primary afferent fibers declines as one moves from distal to proximal areas on the hand (Johansson \& Vallbo, 1979). Overall, the results from the GO task were consistent with these changes in the density of innervation. Table 3 presents the estimates of the density of innervation of both SAI and RA fibers that have been made in humans and monkeys (DarianSmith \& Kenins, 1980; Johansson \& Vallbo, 1979). Both studies showed that the density of innervation for both types of fibers declines substantially as one moves proximally. From these estimates, we calculated the resulting spacing for the SAI receptors for the human data shown in 


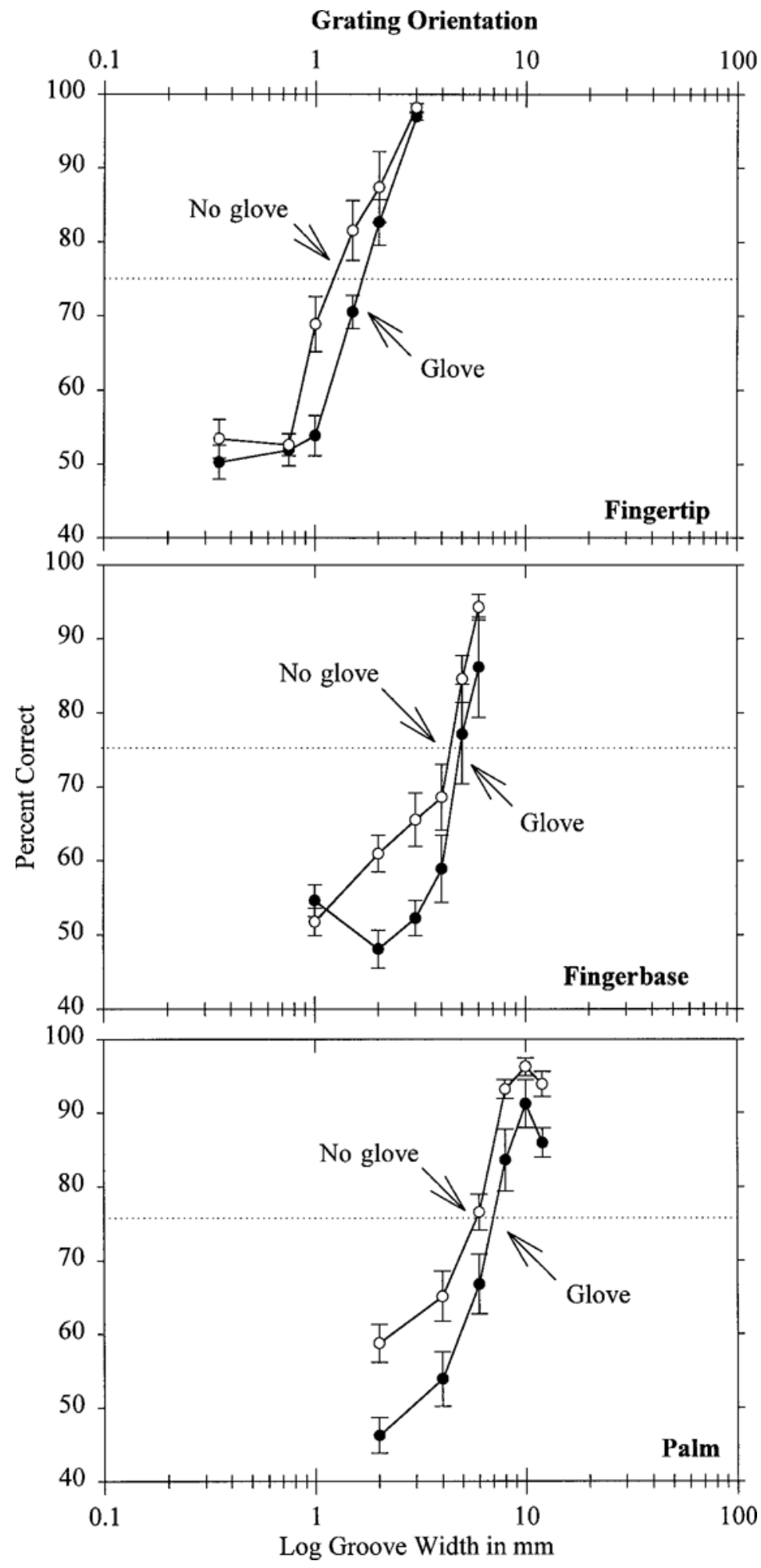

Figure 1. Percent correct as a function of log groove width (in millimeters) at the index fingertip, fingerbase, and palm both without a glove and with a glove for the GO task. Error bars represent \pm 1 standard error of the mean. The dashed line represents $75 \%$ correct performance. 
Table 2

Grating Orientation Thresholds (in Millimeters): $75 \%$ Correct Performance and Percent Increase in Threshold with Glove

\begin{tabular}{lccc}
\hline \multicolumn{1}{c}{ Location } & No Glove & With Glove & \% Increase \\
\hline Distal fingerpad & 1.24 & 1.69 & 36 \\
Proximal fingerpad & 4.35 & 4.93 & 13 \\
Palm & 5.73 & 6.98 & 22 \\
\hline
\end{tabular}

Table 3 for the three locations. There is strong evidence that SAI fibers are responsible for conveying fine spatial information (Johnson \& Hsiao, 1994). The estimated spacing for the fingertip, fingerbase, and palm are 1.2 , 1.8 , and $3.5 \mathrm{~mm}$, respectively. Assuming that spacing of SAI receptors is a major determinant of GO sensitivity, the thresholds for the three sites should be approximately the same as the estimated spacing of the receptors. The thresholds, as shown in Table 2, fit the estimated spacing at the fingertip but are higher than expected at the palm and considerably higher at the fingerbase. These results are consistent with the results of earlier studies (Craig, 1999; Craig \& Lyle, 2001). It has been suggested that spatial acuity is poorer than the estimated density of innervation would suggest because of central limitations in processing (Craig \& Lyle, 2002).

There was a moderate loss of GO sensitivity due to the gloves. With the inconsistent pattern of results from previous studies, it is difficult to say what one ought to expect in the GO task with gloves. Modest changes might go unnoticed, depending on the sensitivity of the measuring technique and the degree to which the measure is related to spatial acuity. In some of the previous studies, subjects were allowed to move their hands, whereas, in the present study, movement was not permitted. If movement were used to detect the orientation of a grating, sensitivity would be much greater, although performance would then be unlikely to reflect strictly spatial mechanisms.

\section{EXPERIMENT 2}

The SG task was used to assess the effect of gloves on sensitivity at the same three sites on the hand as those in Experiment 1. As noted, this task was a variation on gap detection tasks that appear to measure spatial acuity (Johnson \& J. R. Phillips, 1981; Stevens \& Choo, 1996; Stevens \& Patterson, 1995). One aim of Experiment 2 was to see whether gloves would have the same effect on the SG task as they did on the GO task and whether similar changes in sensitivity would be found across sites of stimulation. Whether or not the SG task accurately reflects changes in spatial acuity, it may provide an important measure of tactile functioning: the ability to detect a smooth surface from a grooved surface.

\section{Method}

Subjects. As in Experiment 1, the subjects were students at Indiana University who were paid employees of the laboratory. The number of subjects tested in each condition is given in Table 1 .

Stimuli. For the SG task, the stimuli were the same as those in the GO task, except for an additional smooth contactor (no grooves).
The smooth contactor was identical to the grooved contactors, except that it lacked grooves.

Apparatus. The apparatus was the same as that in the GO task.

Procedure. In the SG task, the subjects were instructed that two different contactors would be used. They were told that one of the contactors was smooth and that the other was grooved. They were then shown the contactors. On each trial, they were to respond either "smooth" or "grooved." The grooved contactor was presented with the grooves oriented in the proximal-distal direction. Both contactors were demonstrated with the subjects observing the presentation. The subjects then received several practice trials in which they kept their eyes closed. As in the GO task, each session began with practice trials with feedback; however, during testing, no feedback was provided. To reduce the possibility that thermal cues might help the subjects discriminate between a smooth contactor and a grooved contactor (Craig, 1999), all contactors were warmed to the approximate temperature of the skin.

The procedures for the SG task on the fingertip, fingerbase, and the palm were similar to those used in the GO task, with the exception that a smooth contactor was used. The contactors were presented to the same locations as those used in Experiment 1. For the fingertip, the same contactor groove widths were used in the SG task as those in the GO task. At the fingerbase and the palm, the subjects showed greater sensitivity in this task than in the GO task. Therefore, the subjects were tested with smaller groove widths than those in Experiment 1. For the fingerbase and the palm, each session started with the first block using a groove width of $6.0 \mathrm{~mm}$. Each successive block of trials tested a smaller groove width: 4, 2, 1.5, 1.0, and $0.75 \mathrm{~mm}$. The contactor presented on each trial (smooth or grooved) was randomly selected.

\section{Results and Discussion}

Figure 2 presents the results from the SG task. As in Figure 1 , percent correct is plotted as a function of the log of the groove width. Table 4 shows the threshold results (75\% correct) and the percentage change in threshold resulting from wearing the gloves. It is clear that gloves had a substantial effect on the performance of this task with thresholds increasing by $100 \%$ to more than $300 \%$. The ANOVA showed that the glove had a significant effect on performance at the fingertip, fingerbase, and palm $[F(1,7)=$ $87.65, F(1,7)=26.81$, and $F(1,9)=41.89$, respectively, $p$ s $<.01]$, and there was a significant effect of groove width at the fingertip, fingerbase, and palm $[F(5,35)=$ $143.83, F(5,35)=34.93$, and $F(5,45)=42.18$, respectively, $p$ s $<.01$ ]. There was also a significant interaction between glove and groove width at the fingertip and at the palm $[F(5,35)=15.94$, and $F(5,45)=4.33$, respectively, $p$ s $<.01]$. As with the GO task, this interaction might reflect the two functions approaching one another as performance became asymptotic.

Table 3

Estimated Density of Innervation Per Square Centimeter

\begin{tabular}{lrrrrr}
\hline & \multicolumn{2}{c}{ Human } & & \multicolumn{2}{c}{ Monkey } \\
\cline { 2 - 3 } \cline { 6 - 7 } \multicolumn{1}{c}{ Location } & RA & SAI & & RA & SAI \\
\hline Distal fingerpad & 140 & 70 & & 178 & 134 \\
Proximal fingerpad & 37 & 30 & & 80 & 46 \\
Palm & 24 & 8 & & n.a. & n.a. \\
\hline
\end{tabular}

Note-The "Human" data were from Johansson and Vallbo (1979). The "Monkey" data were from Darian-Smith and Kenins (1980). RA, rapidly adapting; SAI, slowly adapting Type I; n.a., not available. 


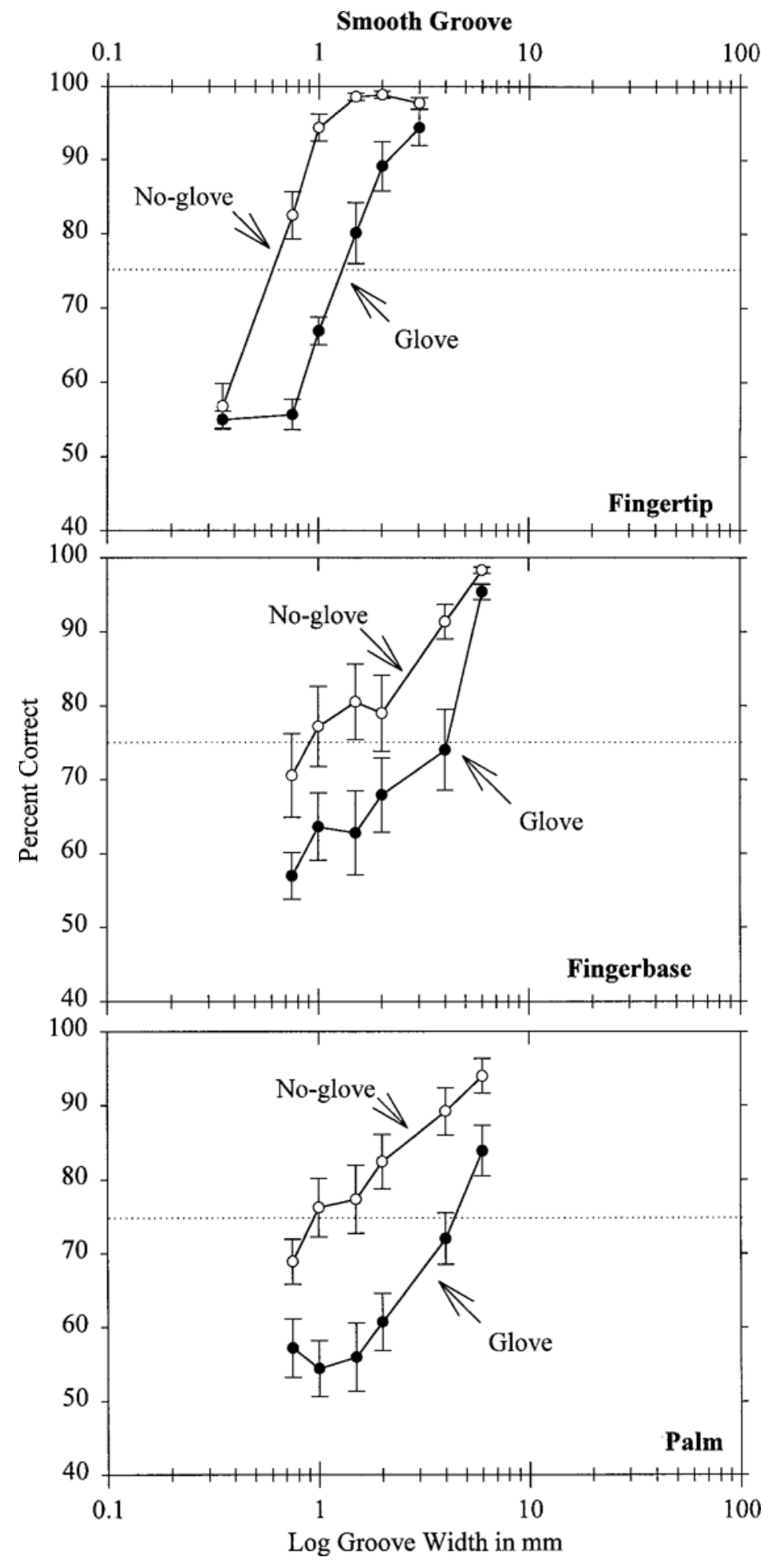

Figure 2. Percent correct as a function of log groove width (in millimeters) at the index fingertip, fingerbase, and palm both without a glove and with a glove for the SG task. Error bars represent \pm 1 standard error of the mean. The dashed line represents $75 \%$ correct performance. 
Table 4

Smooth Groove Thresholds (in Millimeters): $75 \%$ Correct Performance and Percent Increase in Threshold with Glove

\begin{tabular}{lccc}
\hline \multicolumn{1}{c}{ Location } & No Glove & With Glove & \%Increase \\
\hline Distal fingerpad & 0.63 & 1.31 & 107 \\
Proximal fingerpad & 0.92 & 4.09 & 345 \\
Palm & 0.96 & 4.05 & 322 \\
\hline
\end{tabular}

The SG results differ from the GO results in two important ways. First, in the absence of the gloves, there was only a modest change in threshold with location. Second, gloves produced a large reduction in sensitivity. The reduction was 3 to 10 times more than was seen in the GO task. The results without a glove also differed from the results obtained in earlier studies in which subjects discriminated between a smooth surface and one with a gap in it. In the Stevens and Choo (1996) study, the threshold on the fingertip was approximately $0.95 \mathrm{~mm}$, the threshold on the fingerbase was $3.6 \mathrm{~mm}$, and the threshold in the center of the palm was $6.5 \mathrm{~mm}$. These threshold values are similar to the results that we obtained in the GO task but are considerably larger than those obtained in the SG task. The present results were also unlike Johnson and J. R. Phillips's (1981) results. Their threshold for gap detection was the same as the threshold for their GO task, whereas our SG threshold at the fingertip was considerably smaller than the GO threshold. These differences suggest that performance on the SG task is not limited by the spatial resolution of the skin. A possible reason for these differences and the role of intensity cues in both the SG task and in the GO task are considered in the General Discussion section.

\section{EXPERIMENT 3}

Experiment 3 examined GO sensitivity at the fingerbase. The results from Experiment 1 showed a two-limb function for GO at the fingerbase. In Experiment 3, we examined some possible reasons for the shape of this function. A possibility is that subjects are discriminating between the two orientations on the basis of intensity cues. If one orientation led to a more intense percept of the stimulus (e.g., greater roughness) than the other orientation, the task could be done on the basis of how rough or how smooth the contactors felt rather than on spatial cues.

We identified three possible ways that intensive cues could have been used in the GO task on the fingerbase. First, it is possible that a small amount of movement could have been introduced during the testing. As noted, had movement been allowed, the GO thresholds would have been very low. The GO task was designed to minimize any movement on the part of the subject; however, the possibility does exist that movement could have been introduced. Second, it is possible that the skin at the fingerbase has certain mechanical properties that increase sensitivity in one orientation. In a recent study, Wheat and Goodwin (2000) replicated an earlier finding of J. R. Phillips and Johnson (1981a) that, when grooves are oriented parallel to the dermal ridges, both sensitivity and neural responses are heightened relative to when grooves are oriented orthogonal to the dermal ridges. Third, it is possible that, in one of the orientations, more skin is penetrating into the groove, thus providing an intensive cue.

To examine the possibility that intensive cues were being used in the GO task at the fingerbase, we made three sets of measurements. First, we replicated the original GO task without the glove. Second, we tested GO with the contactors at oblique orientations. Third, we measured the depth of penetration of the skin into the groove in both the lateral and the proximal orientations.

\section{Method}

Subjects. As in Experiments 1 and 2, the subjects were students at Indiana University who were paid employees of the laboratory. The number of subjects tested in each condition is given in Table 1. The subjects tested in Experiment 3 had not participated in either Experiment 1 or Experiment 2.

Stimuli. The stimuli for the GO tasks were the same as those in Experiment 1 for the GO task on the fingerbase. For the penetration measurements, we developed a contactor to measure the depth of penetration of the skin into the grooves of the contactor. The contactor groove width was $4 \mathrm{~mm}$. This groove width was close to the GO threshold on the fingerbase. The contactor was identical to the one used in GO testing, with the exception that we machined a groove along the stem of the contactor. A hole was drilled down the center of the shaft and through the bottom of the contactor. A wire was laid in the groove and inserted through the center hole. As the skin pushed into the groove, it displaced the wire that was in the groove and provided a measure of the depth of penetration.

Procedure. The GO procedures were identical to those in the original GO task, with the exception that these measurements were conducted over six sessions, doubling the number of trials per subject. No gloves were used in Experiment 3. All the measurements were made on the fingerbase of the index finger. The first set of measurements was a replication of the GO task from Experiment 1. The second set of measurements differed only in the orientations of the contactors. The orientations were at right angles to one another, upper left and upper right diagonals. In this oblique GO task, subjects indicated the orientation of the contactor by responding "10 o'clock" or "2 o'clock."

All of the subjects who took part in the GO measurements in Experiment 3 also participated in the penetration measurements. For each subject, the penetration of the skin into the groove was measured three times in both the lateral and the proximal orientations. To obtain this measurement, the contactor was allowed to travel freely into the skin in the same manner as in the GO task. As the skin entered the groove, it displaced the wire. The amount of displacement was measured using a microscope with an eyepiece micrometer. This procedure was repeatable with an accuracy of $5 \mu \mathrm{m}$.

\section{Results}

The two GO functions - the replication and the oblique measurements - are presented in Figure 3. As can be seen, the psychometric function from the replicated GO task was very similar to the original psychometric function (Figure 1, middle panel). The thresholds for the original and the replicated functions were 4.35 and $4.15 \mathrm{~mm}$, respectively. The psychometric function from the oblique GO task, however, no longer had the characteristic two-limb function seen in both the original measurements and the 


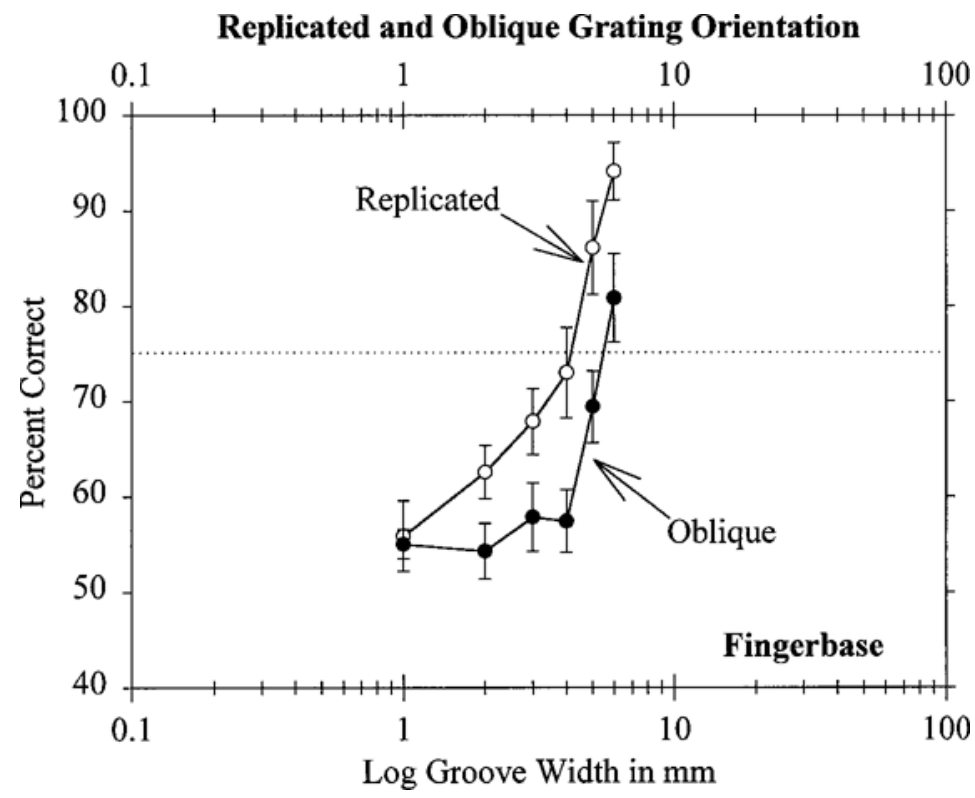

Figure 3. Percent correct as a function of log groove width (in millimeters) at the index finger fingerbase. The GO task replicated function was obtained under conditions similar to those in Experiment 1. The oblique function tested sensitivity to gratings oriented at oblique angles on the finger. Error bars represent \pm 1 standard error of the mean. The dashed line represents $75 \%$ correct performance.

replication. The function dropped in a linear fashion to chance. Also, it should be noted that the slope of the function for the oblique GO task was essentially parallel to the slopes obtained on the fingertip and palm (Figure 1). A repeated measures ANOVA was conducted to see whether there were significant differences between the various conditions. There was not a significant difference between the original GO task and the replicated GO task $[F(1,6)=$ $0.234, p=.646]$. There was, however, a significant difference between the replicated GO task and the oblique GO task $[F(1,6)=28.659, p<.01]$.

The results from the penetration measurements, averaged across subjects, showed that the penetration of skin into the groove in the proximal orientation $[M=0.88 \mathrm{~mm}$, $S D=0.12]$ was approximately twice that seen in the lateral orientation $[M=0.40 \mathrm{~mm}, S D=0.06]$. The difference between the lateral and proximal depths of penetration of skin into the groove was significant, $[t(17)=$ $15.49, p<.001]$.

\section{Discussion}

There are several possible intensive cues that could be affecting the GO function at the fingerbase. First, lateral movement may provide intensive cues in the GO task. When replicating the function, special care was taken to limit the possibility of movement. For lateral movement to produce a differential intensity cue in the GO task, there would have to be greater movement in one orientation than in the other. If there was a similar amount of movement in both orientations, there would be no differential cue for contactor orientation. We saw no evidence for this type of movement. Previous research has shown that minute slip can produce a neural signal that is strong enough to initiate a response (Johansson \& Westling, 1987). This type of movement could provide a cue if subjects could reliably discriminate the direction of slip, although we know of no evidence that such discriminations are possible. We should note that, in the SG task, movement might play a role-a possibility considered in the General Discussion section.

The second possibility is that, like the Wheat and Goodwin (2000) results, our results reflect differential sensitivity in the two orientations. In their study, Wheat and Goodwin found a differential effect of groove orientation on sensitivity at the fingertip in both human subjects and macaque monkeys. Specifically, grooves that were parallel to the dermal ridges produced heightened sensitivity and greater neural responses than did grooves that were orthogonal to the dermal ridges. In a previous study, Craig (1999) found no effect of orientation on performance for the GO task. One possible reason for the difference in results is that, in Wheat and Goodwin's study, the measurements for their human subjects were made at the very tip of the fingerpad. At the tip of the finger, the dermal ridges are fairly uniform in orientation and parallel to the lateralmedial axis of the finger. In Craig's study, measurements were taken $15 \mathrm{~mm}$ proximal to the tip of the finger-an area where the ridges are less uniform in orientation and, thus, an area where one would not expect heightened sen- 
sitivity in one orientation. It seems unlikely that dermal ridges are responsible for the two-limb function at the fingerbase. First, on the fingerbase, dermal ridges are less prominent than on the fingertip. Second, the dermal ridges on the fingerbase do not seem to be oriented in either a consistent proximal-distal or a lateral-medial orientation.

The third possible intensive cue is that of greater conformation of skin into the grooves in one of the orientations than in the other. Although dermal ridges are not prominent on the fingerbase, the underlying anatomical structures might affect performance on the GO task. A review of the anatomy of the finger shows that the finger has a long superficial tendon that lies just below the surface of the skin as it passes through the fingerbase. It may be that the stiffness of the tendon allows less penetration of the skin into the groove in the lateral orientation than in the proximal orientation. Greater penetration of skin into the groove would result in greater curvature of the skin at the edge of the groove. Neurophysiological studies have demonstrated that SAI afferents' firing rates increase with greater degrees of curvature of the skin at an edge (LaMotte \& Srinivasan 1987a, 1987b). The penetration results from Experiment 3 seem to be most closely in agreement with this third, possible intensive cue. The underlying tissue appears to be aligned in the proximal-distal direction in the finger. When the contactor is placed in the lateralmedial orientation, the tendon is perpendicular to the grooves leading to less penetration $(0.40 \mathrm{~mm})$ than in the proximal-distal orientation $(0.88 \mathrm{~mm})$. With less penetration, intensity cues would be reduced in the lateral-medial orientation relative to the proximal-distal orientation, which might lead to greater discriminability.

\section{GENERAL DISCUSSION}

\section{Grating Orientation Without a Glove}

With one notable exception, the slopes of the psychometric functions for the GO task were similar to those obtained in previous studies (Craig, 1999; Craig \& Lyle, 2001; Johnson \& J. R. Phillips, 1981; Van Boven \& Johnson, 1994a). For purposes of comparison, psychometric functions from earlier studies are presented in Figure 4, together with the functions from the present study. As can be seen, nearly all the functions have similar slopes. As Loomis and Lederman (1986) hypothesized, the functions obtained at different sites appear to be similar to one another, differing only by a constant that reflects an overall shift in spatial acuity, and may reflect changes in the density of innervation of the primary afferent fibers. The exception to this rule is the fingerbase without a glove.

\section{Grating Orientation With a Glove}

Gloves had a modest, but significant, effect on GO sensitivity. It has been suggested that to identify correctly the orientation of a grating requires a reasonably intact neural representation of the grating (Van Boven \& Johnson, 1994a, 1994b). This representation depends on the den- sity of innervation, the response of the first-order afferents, and the width of the grooves. There are two reasons, related to the effect of gloves, that performance might decline as groove width decreases. First, the spacing between peripheral afferents becomes greater than the groove width. Second, the curvature of the skin around the edge of the groove is lessened, and fewer peripheral afferents are stimulated (LaMotte \& Srinivasan, 1987a, 1987b). Gloves might thus reduce GO sensitivity in two ways: (1) by, in effect, narrowing the groove width and (2) by reducing the curvature of the skin around the edge of the groove.

To some extent, the glove may simply narrow the effective width of the grooves. The gloves were $0.20 \mathrm{~mm}$ thick. The interposition of the glove could reduce the width of a groove by $0.20 \mathrm{~mm}$ on both edges - that is, reducing the groove width by $0.40 \mathrm{~mm}$. In other words, the threshold with a glove should be $0.40 \mathrm{~mm}$ greater than that without the glove. The differences between the glove and no-glove thresholds at the fingertip were $0.45 \mathrm{~mm}$, at the base of the finger $0.58 \mathrm{~mm}$, and at the palm $1.25 \mathrm{~mm}$. For two of the three locations, the threshold values were close to what would be predicted on the basis of thickness of the gloves alone. For the palm, the large difference between the predicted and obtained thresholds argues against the view that the sole or main effect of the glove is to reduce the groove width. It may be that, as the density of innervation declines from fingertip to fingerbase to palm, the role of the second factor, skin curvature, increases. Reducing the number of fibers responding from a sparsely innervated site may have a greater impact on performance than a similar reduction from a more densely innervated site.

\section{Smooth/Grooved Task Without a Glove}

As noted, the threshold values for the SG task were much lower than the GO thresholds. Also, the SG thresholds did not vary with location, as predicted either by GO sensitivity or by the density of innervation. Psychophysical evidence strongly supports the view that spatial acuity on the fingertip is between 3.5 (the present study) and 4.1 (Craig, 1999) times more sensitive than the fingerbase and is between 4.6 (the present study) to 7.4 (Craig \& Lyle, 2001) times more sensitive than the palm. The modest change in SG threshold from fingertip to fingerbase and palm, approximately 1.5 times that obtained on the fingertip, was well below the changes predicted by spatial acuity measures. These differences suggest that the SG task is not dependent on spatial mechanisms alone. Johnson and J. R. Phillips (1981) suggested that, when the gap size was very small in their gap detection task, subjects were basing their discrimination on intensive information. It may be that the multigrooved contactor used in the present study enhanced intensity cues.

In both tasks, the testing procedure was designed to keep lateral movement between the contactor and the skin to a minimum. If movement were permitted, performance on the SG task would be extremely good. Subjects would easily be able to discriminate between the smooth contac- 


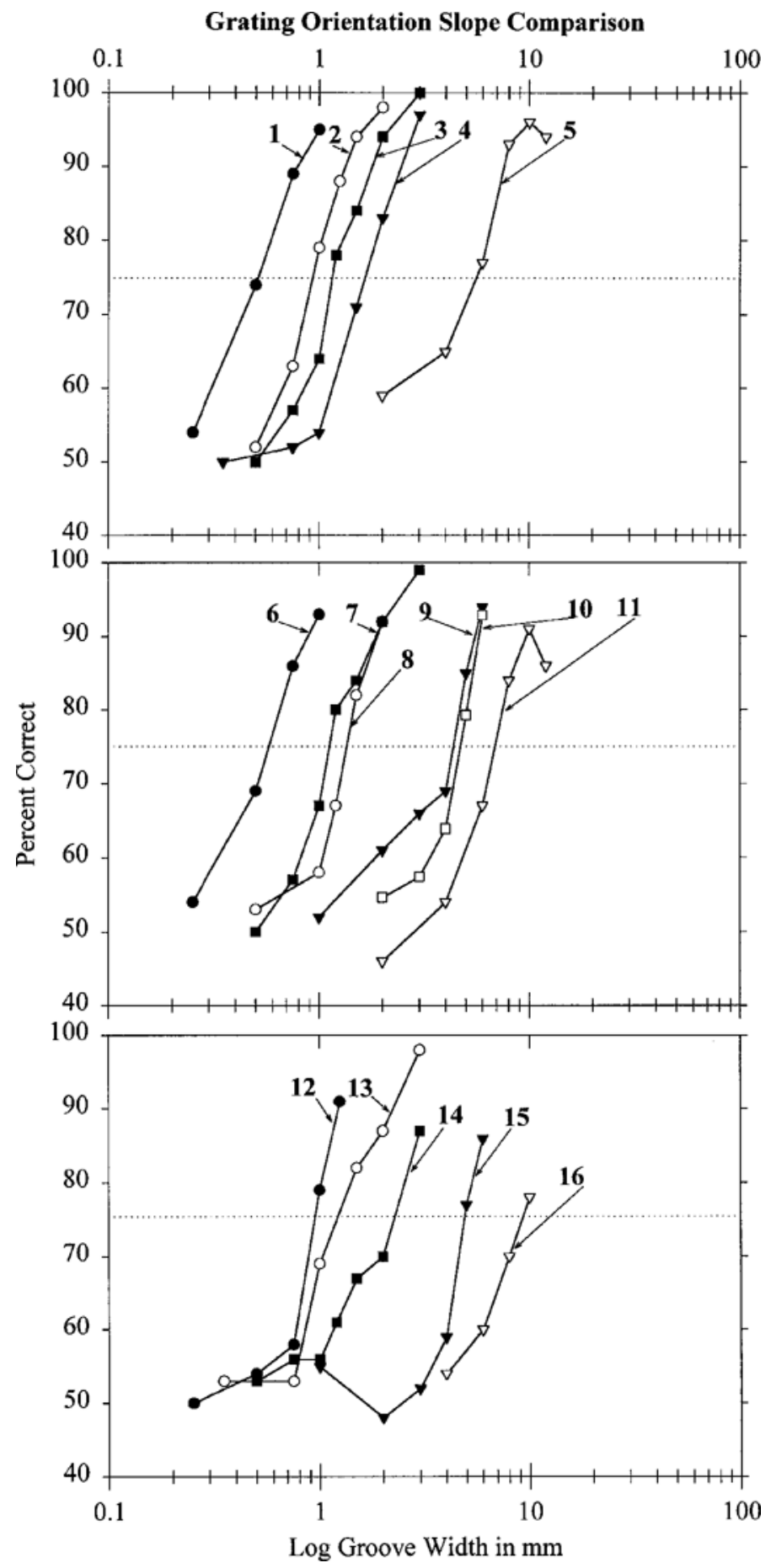

Figure 4. Percent correct as a function of log groove width (in millimeters) for the GO task. The dashed line represents $75 \%$ correct performance. The functions are from previous studies and the present study at several different body loci: (1) lip (Van Boven \& Johnson, 1994a); (2) fingertip (Johnson \& Phillips, 1981); (3) fingertip $5 \mathrm{~mm}$ from tip (Craig, 1999); (4) fingertip with glove (the present study); (5) palm, thenar eminence no glove (the present study). (6) tongue (Van Boven \& Johnson, 1994a); (7) fingertip $15 \mathrm{~mm}$ from tip (Craig, 1999); (8) fingertip (Craig \& Lyle, 2001); (9) fingerbase no glove (the present study); (10) fingerbase (Craig, 1999). (11) palm, thenar eminence with glove (the present study); (12) fingertip (Van Boven \& Johnson, 1994a); (13) fingertip no glove (the present study); (14) fingertip $25 \mathrm{~mm}$ from tip (Craig, 1999); (15) fingerbase with glove (the present study); (16) palm, center (Craig \& Lyle, 2001). 
tor and the finest groove width $(0.35 \mathrm{~mm})$. During testing, the contactor was left in contact with the skin until the subject responded, generally $1-2 \mathrm{sec}$. If the contactor continued to move into the skin during that 1 - to 2 -sec period, the resulting lateral movement of the skin over the edges of the grooves could provide a discriminable signal. Observing the skin and the contactor through a dissecting microscope showed that the contactor remained at a fixed position and then would suddenly move into the skin. This movement was evident with both the smooth contactor and the grooved contactor, but the edges on the grooved contactor would likely result in a stronger signal from the grooved contactor than from the smooth contactor.

\section{Smooth/Grooved With a Glove}

The possibility that movement in the SG task is providing intensive cues may help explain the difference between the SG results with and without a glove. The glove produced a large increase in threshold at all three sites, thresholds that were much closer to the values that would be expected if the task were being done on the basis of spatial information. Some additional measurements showed that the glove decreased the amount of penetration of the contactor into the skin by an average of $27 \%$ and the skin into the groove by $39 \%$. The glove also increases the stiffness of the skin (Bensel, 1993; Brisben et al., 1992; Burke, Watts, \& Wilson, 1989; Cunningham et al., 1992; Jackson et al., 1999; Novak et al., 1999; A. M. Phillips et al., 1997; S. J. Wilson, Sellu, Uy, \& Jaffer, 1996; Woods, Leslie, Drake, \& Edlich, 1996). By reducing the penetration and increasing the stiffness, it is less likely that the skin will move laterally across the edges of the grooves (Taylor \& Lederman, 1975). Reducing intensity cues would reduce sensitivity but permit the discrimination to be done on the basis of spatial information.

As noted, the contactors in the SG task were warmed to approximate skin temperature to avoid thermal cues in discriminating between the smooth and grooved contactors. Craig (1999) found no difference in sensitivity in an SG task, whether or not the contactors were warmed-a result that suggests that subjects were not using thermal cues. Although it is unlikely that thermal cues were being used in the present SG task, the substantial increase in threshold with the latex gloves, a possible thermal insulator, was consistent with the use of thermal cues.

\section{Implications for Testing Sensitivity With Gloves}

How well do the changes in performance, as revealed by the GO and SG tasks, reflect the altered sensitivity that people report while using gloves? A previous result suggests that GO is highly correlated with subjective reports of altered sensitivity (Van Boven \& Johnson, 1994b). It was reported that, out of 11 measures of tactile and thermal sensitivity, the results from only the GO task and warmth measures correlated highly with subjects' perceived sense of normal tactile sensitivity. These results would imply that, to the extent that self-reports of perceived losses in sensitivity with gloves are modest, the GO measure might be a good measure of this loss. The altered sense of touch with a glove may reflect the more or less constant loss of spatial sensitivity across sites. To the extent that self-reports of perceived losses in sensitivity with gloves are large, then the SG task may be a better reflection of this perception. Also, the perception of altered sensitivity may reflect not only an absolute loss of sensitivity but also a relative loss of sensitivity across sites. In the absence of gloves, we seem to take little note of the large differences in sensitivity as different parts of the hand come in contact with an object. We must be able to "normalize" the input from different sites, so that we perceive a uniform object. Gloves may distort this normalization process and contribute to the perception of altered sensitivity.

\section{REFERENCES}

BENSEL, C. K. (1993). The effects of various thicknesses of chemical protective gloves on manual dexterity. Ergonomics, 36, 687-696.

Brisben, A. J., Hsiao, S. S., Looft, F. J., \& Johnson, K. O. (1992). Tactile recognition through intermediate surfaces. Society for Neuroscience Abstracts, 18, 1545.

Burke, F. J., Watts, D. C., \& Wilson, N. H. (1989). Some physical factors influencing tactile perception with disposable non-sterile gloves. Journal of Dentistry, 17, 72-76.

Craig, J. C. (1999). Grating orientation as a measure of tactile spatial acuity. Somatosensory \& Motor Research, 16, 197-206.

Craig, J. C., \& Johnson, K. O. (2000). The two-point threshold: Not a measure of tactile spatial resolution. Current Directions in Psychological Science, 9, 29-32.

Craig, J. C., \& Kisner, J. M. (1998). Factors affecting tactile spatial acuity. Somatosensory \& Motor Research, 15, 29-45.

Craig, J. C., \& LyLe, K. B. (2001). A comparison of tactile spatial sensitivity on the palm and fingerpad. Perception \& Psychophysics, 63, 337-347.

Craig, J. C., \& Lyle, K. B. (2002). A correction and a comment on Craig and Lyle (2001). Perception \& Psychophysics, 64, 504-506.

Cunningham, J. L., Delargy, S. M., \& Warnock, C. M. (1992). Glove wearing in Northern Ireland and an assessment of the loss of tactile perception. Journal of the Irish Dental Association, 38, 12-14.

Darian-Smith, I., \& Kenins, P. (1980). Innervation density of mechanoreceptive fibers supplying glabrous skin of the monkey's index finger. Journal of Physiology, 309, 147-155.

Essock, E. A., Krebs, W. K., \& Prather, J. R. (1997). Superior sensitivity for tactile stimuli oriented proximally-distally on the finger: Implications for mixed class 1 and class 2 anisotropies. Journal of Experimental Psychology: Human Perception \& Performance, 23, 515-527.

Heller, M. A., \& Mitchell, B. Y. (1985). Helping new braille readers: Effects of spacing, finger locus, and gloves. Perceptual \& Motor Skills, 61, 363-369.

Jackson, E. M., Neal, J. G., Williams, F. M., Stern, C. A., Suber, F., ThaCKer, J. G., \& EDLICH, R. F. (1999). Biomechanical performance of orthopedic gloves. Journal of Biomedical Materials Research, 48, 193-198.

Johansson, R. S., \& Vallbo, A. B. (1979). Tactile sensibility in the human hand: Relative and absolute densities of four types of mechanoreceptive units in glabrous skin. Journal of Physiology, 286, 283-300.

Johansson, R. S., \& Westling, G. (1987). Signals in tactile afferents from the fingers eliciting adaptive motor responses during precision grip. Experimental Brain Research, 66, 141-154.

JoHnSON, K. O., \& HSIAO, S. S. (1992). Neural mechanisms of tactual form and texture perception. Annual Review of Neuroscience, 15, 227 250 . 
Johnson, K. O., \& Hsiao, S. S. (1994). Evaluation of the relative roles of slowly and rapidly adapting afferent fibers in roughness perception. Canadian Journal of Physiology \& Pharmacology, 72, 488-497.

Johnson, K. O., \& Phillips, J. R. (1981). Tactile spatial resolution: I. Two-point discrimination, gap detection, grating resolution, and letter recognition. Journal of Neurophysiology, 46, 1177-1191.

Johnson, K. O., Van Boven, R. W., \& Hsiao, S. S. (1994). The perception of two points is not the spatial resolution threshold. In J. Boivie, P. Hansson, \& U. Lindblom (Eds.), Touch, temperature, and pain in health and disease: Mechanisms and assessments (11th ed., pp. 389-404). Seattle: IASP.

KLATZKy, R. L., \& Lederman, S. J. (1999). Tactile roughness perception with a rigid link interposed between skin and surface. Perception \& Psychophysics, 61, 591-607.

LaMotte, R. H., \& Srinivasan, M. A. (1987a). Tactile discrimination of shape: Responses of slowly adapting mechanoreceptor afferents to a step stroked across the monkey fingerpad. Journal of Neuroscience, 7, 1655-1671.

LaMotte, R. H., \& Srinivasan, M. A. (1987b). Tactile discrimination of shape: Responses of rapidly adapting mechanoreceptive afferents to a step stroked across the monkey fingerpad. Journal of Neuroscience, 7, 1672-1681.

Loomis, J. M., \& Lederman, S. J. (1986). Tactual perception. In K. R. Boff, L. Kaufman, \& J. P. Thomas (Eds.), Handbook of perception and human performance (Vol. 31, pp. 1-41). New York: Wiley.

Nelson, J. B., \& Mital, A. (1995). An ergonomic evaluation of dexterity and tactility with increase in examination/surgical glove thickness. Ergonomics, 38, 723-733.

Novak, C. B., Patterson, J. M., \& Mackinnon, S. E. (1999). Evaluation of hand sensibility with single and double latex gloves. Plastic \& Reconstructive Surgery, 103, 128-131.

Patel, J., Essick, G. K., \& Kelly, D. G. (1997). Utility of square-wave gratings to assess perioral spatial acuity. Journal of Oral \& Maxillofacial Surgery, 55, 593-601.

Phillips, A. M., Birch, N. C., \& Ribbans, W. J. (1997). Protective gloves for use in high-risk patients: How much do they affect the dexterity of the surgeon? Annals of the Royal College of Surgeons of England, 79, 124-127.

Phillips, J. R., \& Johnson, K. O. (1981a). Tactile spatial resolution: II. Neural representation of bars, edges, and gratings in monkey primary afferents. Journal of Neurophysiology, 46, 1192-1203.

Phillips, J. R. \& Johnson, K. O. (1981b). Tactile spatial resolution: III. A continuum mechanics model of skin predicting mechanoreceptor responses to bars, edges, and gratings. Journal of Neurophysiology, 46, 1204-1225.
Sathian, K., \& Zangaladze, A. (1996). Tactile spatial acuity at the human fingertip and lip: Bilateral symmetry and interdigit variability. Neurology, 46, 1464-1466.

Stevens, J. C. (1990). Perceived roughness as a function of body locus. Perception \& Psychophysics, 47, 298-304.

Stevens, J. C., \& Choo, K. K. (1996). Spatial acuity of the body surface over the life span. Somatosensory \& Motor Research, 13, 153166.

Stevens, J. C., \& Patterson, M. Q. (1995). Dimensions of spatial acuity in the touch sense: Changes over the life span. Somatosensory \& Motor Research, 12, 29-47.

TAY LOR, M. M., \& Lederman, S. J. (1975). Tactile roughness of grooved surfaces: A model and the effect of friction. Perception \& Psychophysics, 17, 23-36.

Thompson, P. B., \& LamberT, J. V. (1995). Touch sensitivity through latex examination gloves. Journal of General Psychology, 122, 47-58.

VAN Boven, R. W., \& Johnson, K. O. (1994a). The limit of tactile spatial resolution in humans: Grating orientation discrimination at the lip, tongue, and finger. Neurology, 44, 2361-2366.

VAn Boven, R. W., \& Johnson, K. O. (1994b). A psychophysical study of the mechanisms of sensory recovery following nerve injury in humans. Brain, 117 (Pt. 1), 149-167.

Webb, J. M., \& Pentlow, B. D. (1993). Double gloving and surgical technique. Annals of the Royal College of Surgeons of England, 75, 291-292.

Wheat, H. E., \& Goodwin, A. W. (2000). Tactile discrimination of gaps by slowly adapting afferents: Effects of population parameters and anisotropy in the fingerpad. Journal of Neurophysiology, 84, 14301444.

Wilson, M. P., Gound, S., TishK, M., \& Feil, P. (1986). Gloved versus ungloved dental hygiene clinicians: A comparison of tactile discrimination. Dental Hygiene, 60, 310-315.

Wilson, S. J., Sellu, D., Uy, A., \& Jaffer, M. A. (1996). Subjective effects of double gloves on surgical performance. Annals of the Royal College of Surgeons of England, 78, 20-22.

Woods, J. A., Leslie, L. F., Drake, D. B., \& Edlich, R. F. (1996). Effect of puncture resistant surgical gloves, finger guards, and glove liners on cutaneous sensibility and surgical psychomotor skills. Journal of Biomedical Materials Research, 33, 47-51.

(Manuscript received June 13, 2001; revision accepted for publication December 13, 2001.) 